\title{
Grid storage in LV networks - An appropriate solution to avoid network limitations in high RES scenarios
}

\author{
Mihai Sanduleac \\ Mihaela Albu \\ University Politehnica of Bucharest, \\ Bucharest, Romania \\ mihai.sanduleac@gmail.com \\ albu@ieee.org
}

\author{
Dorel Stanescu \\ Metering Department \\ SDEE Transilvania Sud \\ Brasov, Romania \\ dorel.stanescu@electrica-distributie.ro
}

\author{
Carmen Stanescu \\ Metering Branch OMEPA \\ Transelectrica SA \\ Sibiu, Romania \\ carmen.stanescu@transelectrica.ro
}

\begin{abstract}
Low voltage networks are expected to see massive transformations in the next decades. The aim for a carbon neutral economy asks for high level penetration of renewables, where $P V$ production in LV networks is an important part of the overall solution. The RES high penetration becomes however also a threat for grid operation, through challenges such as keeping voltage level in the accepted band, avoiding grid congestion and reverse power flow or ensuring an acceptable low level of energy losses. The paper analyses specific scenarios and suggests measures to address these limitations, by comparing business as usual operation with more advanced microgrid operation and the use of grid-side storage.
\end{abstract}

Index Terms-prosumer, active distribution grid, RES, storage

\section{INTRODUCTION}

Low voltage networks are expected to see massive transformations in the next decades. The price drop and new technologies of PV panels makes now possible to produce in cost effective terms more and more energy locally, fed directly in the low voltage (LV) network. These LV grids become not only active distribution networks but can be operated also as microgrids (MG) to support local resilience, off-line operation and can reach higher efficiency energy transfer within the MG contour. However, it is well known that in a "business as usual" (BaU) paradigm, excessive PV production also brings high challenges to the grid operator, in terms of keeping voltage level in the accepted band of operation $\left(\mathrm{U}_{\mathrm{N}} \pm 10 \%\right.$ for low voltage), avoiding grid congestion on lines and on the $\mathrm{MV} / \mathrm{LV}$ transformer, avoiding reverse power flow in the transformer and keeping acceptable level of losses in the LV grid.

Various studies [1-9] and regulatory initiatives address these challenges, however still with low impact on the microgrid design and operation, as part of the distribution system operators (DSOs) management.

New grid storage services, as well as the so called "storage behind the meter" are considered solutions to mitigate these issues. In [11] and [12] are analyzed the benefits of having prosumers with no active power injection back to the main grid, enabled by appropriate design of the PV production, of local battery storage and of specific local energy management system. Such solution allows that the main grid is operated in a $\mathrm{BaU}$ paradigm (prosumers are seen as consumer, due to no active power generation sent back to the grid), while prosumers with high self-consumption have also an enhanced resilience and energy efficiency.

Microgrid as a complex entity, might be seen as a vehicle for a high penetration of the renewables, and therefore able to contribute in addressing climate change countermeasures. The EU new average target of $32 \%$ renewable energy by 2030 [12], in a mix of electricity, heating/cooling and mobility, may ask for a higher rate on clean electricity only, somewhere between 50 and $60 \%$, as today technology readiness level (TRL) is higher in the electricity domain rather than in transportation or in heating/cooling.

We are considering in this paper that at least $30 \%$ of RESbased electricity shall be provided locally, i.e. being associated with low power RES based generation, suitable for injecting the electricity in LV micro-grids, while the remaining share of renewables can be provided from bulk RES-based production placed at higher voltage levels, connected at medium voltage (MV) and high voltage (HV) networks.

Recently issued regulatory initiatives (like, for example, [13] in Romania) will encourage more domestic users to install PV panels on their home's rooftops, changing their contractual relation with the DSO. The DSO must prepare itself for changes in the power flow in LV network a subsequently to changes in planning and operation.

RES penetration in LV networks is also very important regarding Total Losses evolution. For example, one needs to estimate the amount of DG production that does not increase the technical losses in LV network.

On the other hand, with prosumers having very low or no energy exchanges with the network (implying that they have storage on their premise), the meter accuracy will be very important when commercial losses are needed to be taken into account. 


\section{Test CASE. ThE ANALySED GRID}

In order to assess the challenges and the feasibility for a RES share of more than $30 \%$ in a local LV grid, we used a test grid named UPB-TEST01A-LV, which has been chosen to put in value the scope and to have in certain periods and configurations situations bringing grid limits violations, which need to be addressed by operational measures during a full day time.

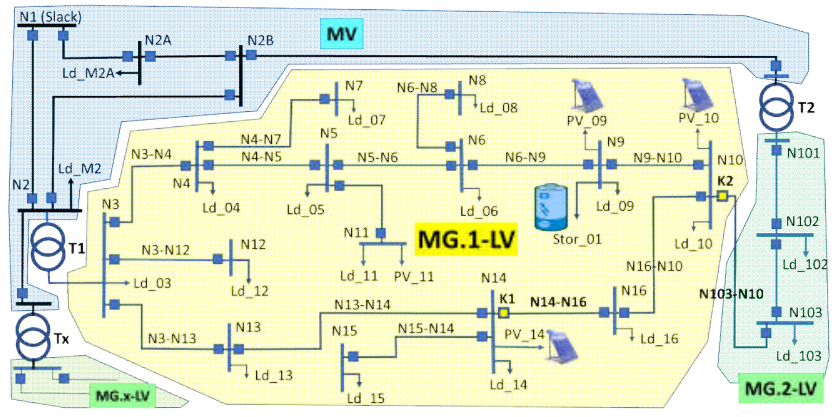

Fig. 1. Analysed test grid

Fig. 1 presents the considered test grid, which includes the microgrid MG.1-LV, which is the focus of our study. This microgrid is supplied from medium voltage through the transformer T1. An additional LV microgrids supplied by transformer T2 is represented in a simplified way, and is basis for a more future-oriented topology change option, as it is possible a "horizontal connection" between MG.1 and MG.2 through the LV line N103-N10, normally open, as its breaker $\mathrm{K} 2$ is also open. The line N103-N10 acts like a tie-line between the MGs, which may be a possible type of connection intelligent microgrids of the future.

Moreover, the classic radial topology of the LV networks is challenged as well through an operational loop connection between two branches, allowed by the additional loop line N14-N16, which is in normal state disconnected, as having the breaker K1 open.

The main MG.1 microgrid is supplied from three gridconnected PV generators (nodes N9, N10 and N14), which are supposed to be part of community energy efforts, to be shared eventually by all local grid users. A storage resource connected to node N10 may be used for the purpose of improving MG operation.

The main characteristics of loads, generators and storage means of the MG.1 are presented in Table 1. The figures show nominal powers, however during a day these are multiplied by time-interval based profiles factors (potentially different values for each hour) which are in the range $[0,1]$, making the full day profile to take values between 0 and $\mathrm{P}_{\mathrm{N}}$ or $\mathrm{Q}_{\mathrm{N}}$.

To be noted that, while the MV node N1 acts as slack node for the power flows, the node N3 on the LV part of the transformer $\mathrm{T} 1$ is also acting as a node of stability, which brings also the so-called grid forming functionality to the microgrid.
TABLE I. MG.1-LV: NODE PARAMETERS

\begin{tabular}{|c|c|c|c|c|}
\hline Name & Load & PV & Storage & Obs. \\
\hline N3 & $12+j 0$ & - & - & $\begin{array}{c}\text { MG.1 supply } \\
\text { through T1 }\end{array}$ \\
\hline N4 & $36+j 4.5$ & - & - & - \\
\hline N5 & $42+j 0$ & - & - & - \\
\hline N6 & $45+j 9$ & - & - & - \\
\hline N7 & $30+j 6$ & - & - & - \\
\hline N8 & $15+j 6$ & - & - & - \\
\hline N9 & $30+j 6$ & $200+j 0$ & $\begin{array}{c}0+j 0 \\
100+j 0\end{array}$ & - \\
\hline N10 & $30+j 6$ & $100+j 0$ & - & $\begin{array}{l}\text { Connection } \\
\text { to MG.2: K2 }\end{array}$ \\
\hline N11 & $12+j 3$ & - & - & - \\
\hline $\mathrm{N} 12$ & $60+j 3$ & - & - & - \\
\hline $\mathrm{N} 13$ & $90+j 3$ & - & - & - \\
\hline N14 & $90+j 3$ & $6+j 0$ & - & $\begin{array}{c}\text { Make loop in } \\
\text { MG.1: K1 }\end{array}$ \\
\hline N15 & $15+j 3$ & - & - & - \\
\hline N16 & $10+j 0$ & - & - & - \\
\hline
\end{tabular}

In order to assess the MG.1 functionality, six different scenarios have been chosen:

- Scenario 1: Business as usual (BaU), LV network with radial supply, meaning that there is no LV loop (K1 open) and no connection with another LV network (K2 open); moreover, no electricity storage is used (storage resource in N9 has always $0+j 0$ power contribution);

- Scenario 2: More flexibility in the LV MG1, by establishing an LV loop with K1 closed and line N14N16 allowing power exchange between main branches; there is still no connection with another LV network (K2 open); no electricity storage is used (storage resource in $\mathrm{N} 9$ has always $0+\mathrm{j} 0$ power contribution);

- Scenario 3: The LV network works with radial supply, (no LV loop, K1 open), however a connection with the second microgrid MG.2 is enabled by closing K2, thus the line N103-N10 is exchanging power between the two MGs; no electricity storage is used;

- Scenario 4: The LV network works with an LV loop (K1 closed), and with a connection with the second microgrid MG.2 (K2 closed); no electricity storage is used;

- Scenario 1.Bat: Business as usual: radial supply, (no LV loop, K1 open), no connection with another MG, K2 open; the difference from Scenario 1 is that the storage resource connected in $\mathrm{N} 9$ has a maximal power of $100 \mathrm{~kW}$, ideally accessed any time (no restrictions on the capacity) and has a power contribution according to a schedule which counts on 
charging when there is excess power and discharging when it is excess consumption;

- Scenario 2.Bat: LV loop (K1 open), no connection with another $\mathrm{MG}, \mathrm{K} 2$ open; the difference from Scenario 2 is that the storage resource connected in N9 has a nominal power of $100 \mathrm{~kW}$ and has a power contribution according to a schedule to charge during excess power and discharge during excess consumption.

The six scenarios are tested on a whole day basis, using hourly profiles for consumption, PV production and storage energy exchange with the grid. Essential aspects are considered for the grid operation, such as voltage level in critical nodes, power exchange in the $\mathrm{MV} / \mathrm{LV}$ transformer, total microgrid losses and RES share in the energy mix.

\section{SimUlATIONS AND RESUlTS}

For assessing the grid operation in all 6 scenarios, it has been used an OpenDSS load-flow application. OpenDSS is an open-source tool developed by EPRI [14-16] which is already widely used by American utilities and in different IEEE studies [17-19].

In addition, a graphical user interface (GUI) with enhanced functionalities, named GridMonK (Grid Monitoring and control Knowledge), has been used to handle more complex operations and reporting, by using in background the OpenDSS engine. GridMonK is also an open-source application which can be found at [20]. GridMonK is able to show grid objects, with specific parameters and with associated results after invoking the OpenDSS engine, while operational changes can be done online, such as opening or closing breakers, which change accordingly the topology for the load-flow calculation. Moreover, an evolving set of additional calculations can be made with the load-flow results, in order to provide full data reporting in csv files, for further analysis with external tools such as Excel.

The daily (24 hours) simulation is made in all six scenarios, by using similar profiles for all loads, but with different nominal values according to Table 1 . The presentation of the simulations and the assessment of the results is divided in categories: impact on voltage level, power flow on transformers, grid losses and RES share.

\section{A. Voltage level in critical nodes}

The critical nodes are those which are distant from the T1 $\mathrm{MV} / \mathrm{LV}$ transformer acting as a supply point, where also bulk PV production of $200 \mathrm{~kW}$ (node N9) and $90 \mathrm{KW}$ (node N10) are present.

We have chosen for our detailed analysis the node N16, which is a consumption point in an electrically "distant" region from the microgrid supply point given by $\mathrm{N} 3$ node (LV part of the transformer T1). Figure 2 shows the voltage evolution over 24 hours in node N16 in each of the scenarios 1 to 4 , as node N16 has been found the most critical in the studied network.

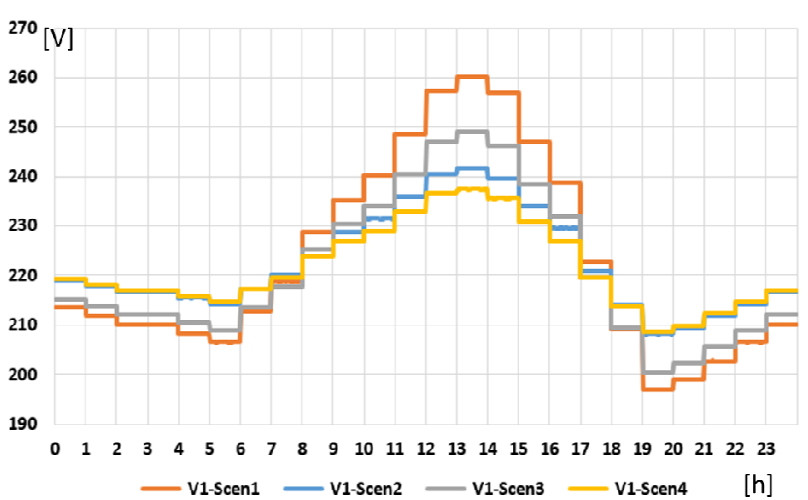

Fig. 2. Voltage levels in the critical node N16, in scenarios without using grid storage (Scenarios 1, 2, 3 and 4)

It can be observed that the scenarios with higher connectivity (more meshed network - meaning Scenario 2 with LV loop and Scenario 4, with LV loop plus intermicrogrid connection) bring a better voltage evolution. The two scenarios with lower connectivity (Scenario 1 with $\mathrm{BaU}$ radial distribution and scenario 3 with added microgrid interchange) are both violating voltage limits $U_{N} \pm 10 \%$ and need additional measures (tap position change on $\mathrm{T} 1$, reactive power control etc.). The worst case is Scenario $1-\mathrm{BaU}$, which explains why grid operators are so much afraid of massive RES penetration in their distribution grid, when using classic operation approach.

Figure 3 presents the voltage level variation in scenarios 1 and 2 , compared with the same situations when a storage device of $100 \mathrm{~kW}$ is acting in node $\mathrm{N} 9$.

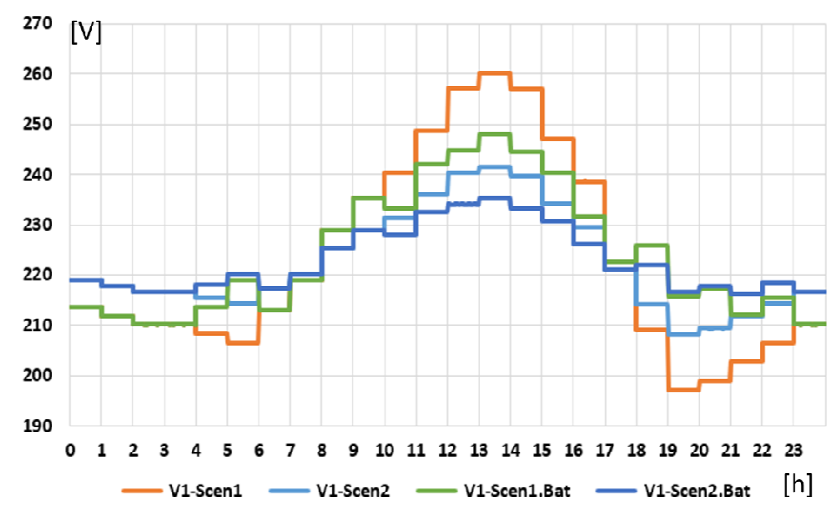

Fig. 3. Voltage levels in the critical node N16, comparing scenarios 1 and 2 with 1.Bat and 2.Bat, where grid battery is also used

One can see that the presence of a $100 \mathrm{~kW}$ storage device (battery energy storage system - BESS) is highly improving the operation of the microgrid without any additional connection with another MG. A straightforward simple solution is solving "like a miracle" the headaches of grid operators, if appropriate schedule is applied for the BESS. Fig. 4 shows the BESS schedule superposed with the previous voltage variation in the most difficult Scenario 1 (BaU), suggesting that a simple algorithm of charging during excess RES production (with high voltage) and discharging during 
excess consumption (with low voltage) has a very good impact to meet grid voltage constrains.

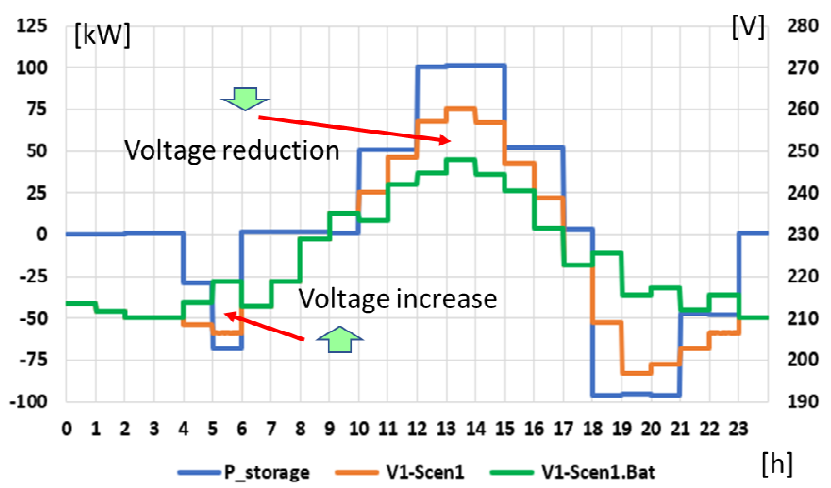

Fig. 4. BESS schedule versus Scenario 1 voltage variation in critical node

It can be seen that the presence of BESS has a positive impact on the voltage level. To be noticed that the charging and discharging energies should be equal over the whole 24 hours' time period (simplified approach, without considering battery losses - as used in our simulations), while a more refined approach should consider the losses as well.

\section{B. Power flow on $M V / L V$ transformer and on $L V$ inter- microgrid line}

Another drawback of high RES penetration is the power flow which acts bidirectionally, including a reverse power on the MV/LV transformer. Fig. 5 shows the transformer power flow in scenarios 1 to 4 .

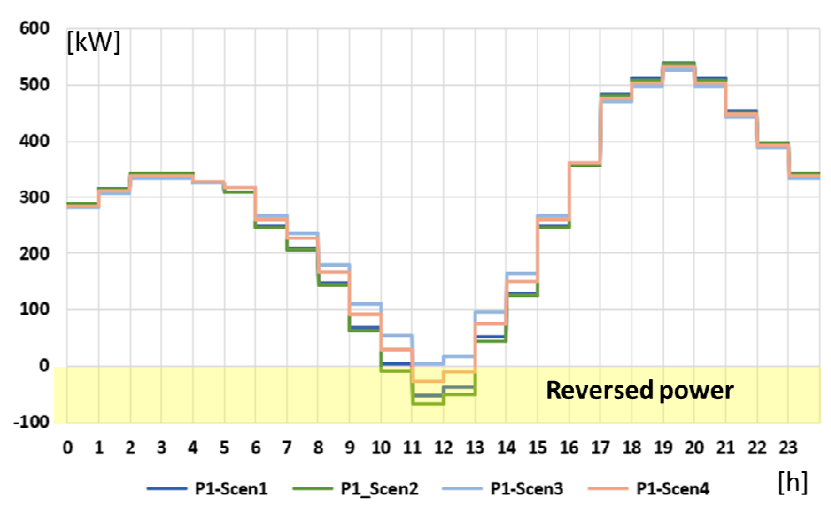

Fig. 5. Power evolution of transformer T1 in scenarios 1,2,3 and 4

It can be seen that only in Scenario 3 there is, at the limit, no reverse power on the transformer, which is due to the fact that some PV power is evacuated in MG.2. All scenarios show high power variation, from nearly the nominal $\mathrm{T} 1 \operatorname{power}\left(\mathrm{S}_{\mathrm{N}}=\right.$ $630 \mathrm{kVA}$ ) to zero or reverse power, which show all a different way of grid operation comparing with the situation without RES penetration.

Fig. 6 shows that activation of BESS in scenarios 1.Bat and 2.Bat bring important changes in the transformer power evolution, by avoiding completely the reverse power and also is reducing the peak power during the evening.

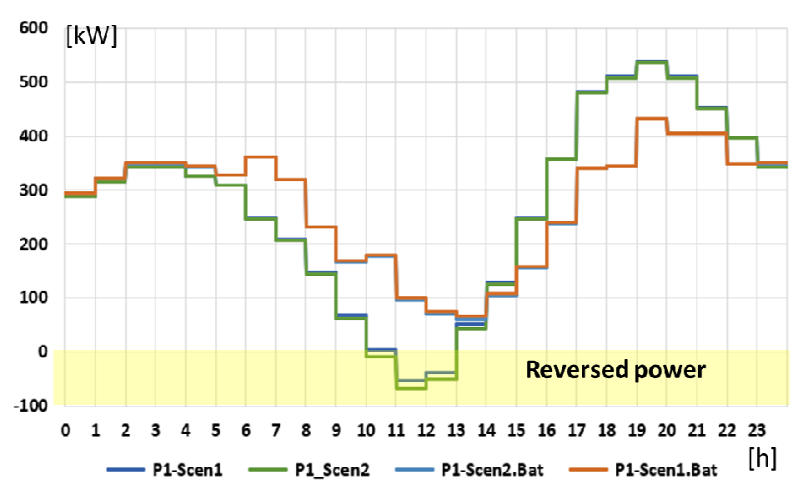

Fig. 6. Comparison on power evolution of transformer $\mathrm{T} 1$ in scenarios 1,2, 1.Bat and 2.Bat

In the latest Figure one can see that the power evolution between around -50 to $+550 \mathrm{~kW}(600 \mathrm{~kW}$ excursion band) is reduced in case of BESS usage to a band between +50 to +450 $\mathrm{kW}$, meaning 400 excursion band, a 33\% reduction compared with no BESS situation. This transformer load profile is much more stable and predictable, and asks for less ancillary services at transmission level, in order to mitigate grid balancing, with impact as well on balancing services cost.

\section{RES share}

One main reason for the high dynamics in the transformer load profile is the variability of RES production, which brings different RES share over the day. Fig. 7 shows the daily evolution of the RES share for our studied scenarios.

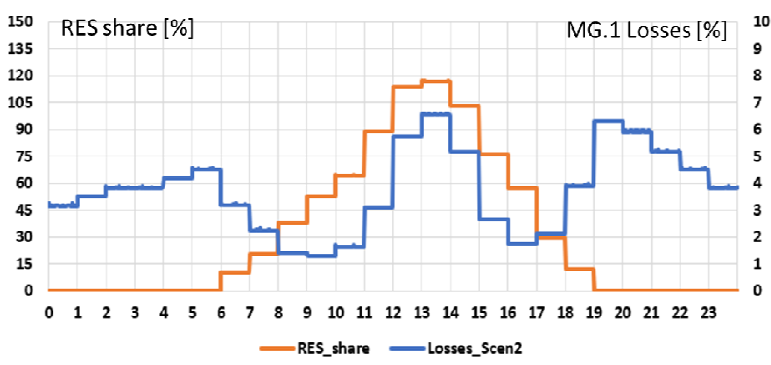

Fig. 7. RES share, superposed by Scenario 2 losses

The RES share evolution is superposed by the microgrid losses evolution during the day, in order to assess the link between them. It can be easily seen the highest losses occur during the excessive PV production during the daytime. To be noted that on a whole day basis, the RES share average is $32 \%$ of the MG. 1 consumption (local RES is producing 32\% of consumed energy over a day in the studied microgrid), which fits the chosen conditions (at least $30 \%$ of power system needed RES to be produced in LV networks).

\section{Grid losses}

While in the previous sub-chapter it has been already presented a coupling between losses and RES share, we made a comprehensive assessment on the losses evolution for all scenarios. 
Fig. 8 shows the losses evolution over the whole day for all scenarios without BESS (scenario 1 to 4), while Fig. 9 shows the impact of the BESS in scenario 1 and 2 when BESS is used: Scenarios 1.Bat and 2.Bat.

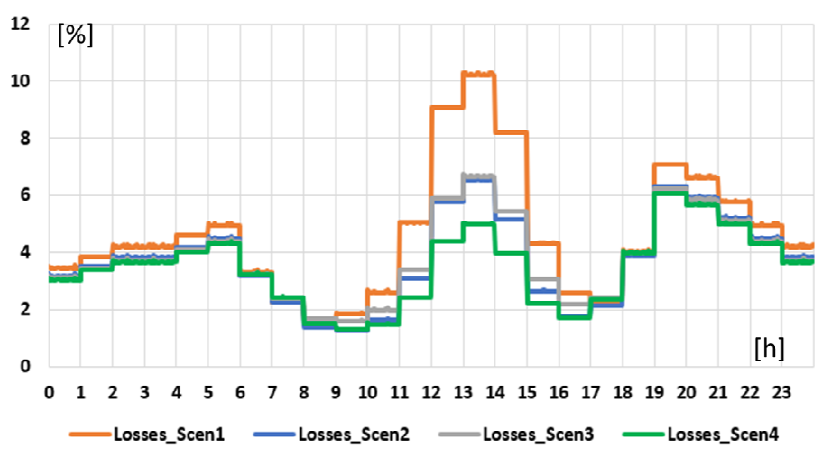

Fig. 8. Comparison on microgrid losses evolution in scenarios 1,2,3,4

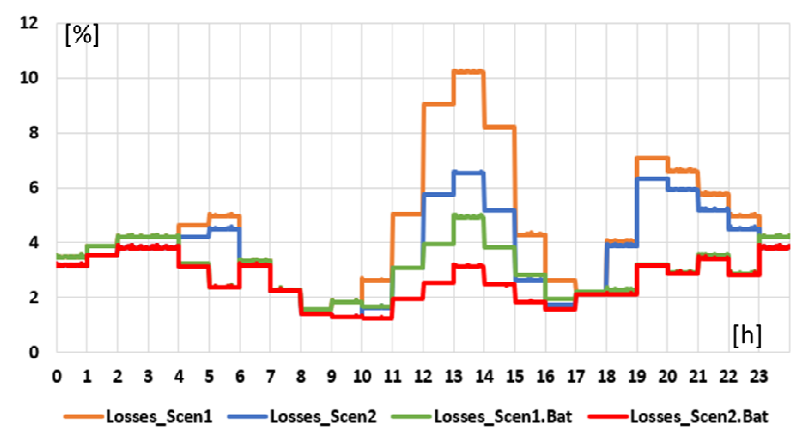

Fig. 9. Comparison on microgrid losses evolution in scenarios 1,2 compared with BESS based usage in scenarios 1.Bat and 2.Bat

Fig. 9 shows high effectiveness in reducing the MG losses when using BESS with an appropriate schedule for charging and discharging.

One can see the relatively odd situation that lowest losses are between hours 9 and 11 and then in the afternoon between hours 16 and 18. This situation shows how counterintuitive become the grid parameters evolution in a network initially designed for one-way power flow, which is evolving towards a grid with local production which may exceed the local consumption in some day periods. In order to better understand this situation, additional assessment has been performed.

Figure 10 shows the evolution of total consumption, total production, their difference and total losses for Scenario 1.

The green line of Figure 10 is practically one shape of the famous "duck-chart" of CAISO [21], where the evening ramp of power from the main grid is very high, while load flows have important changes of direction, which bring also unexpected changes in the total power losses comparing with the traditional experience.

We can see in the Figure that the periods of lowest losses occur in the morning and evening at a similar value of the $\mathrm{P}_{\mathrm{CONS}}+\mathrm{P}_{\mathrm{PV}}$ green line evolution, in the 9-11 and 16-18 time periods.

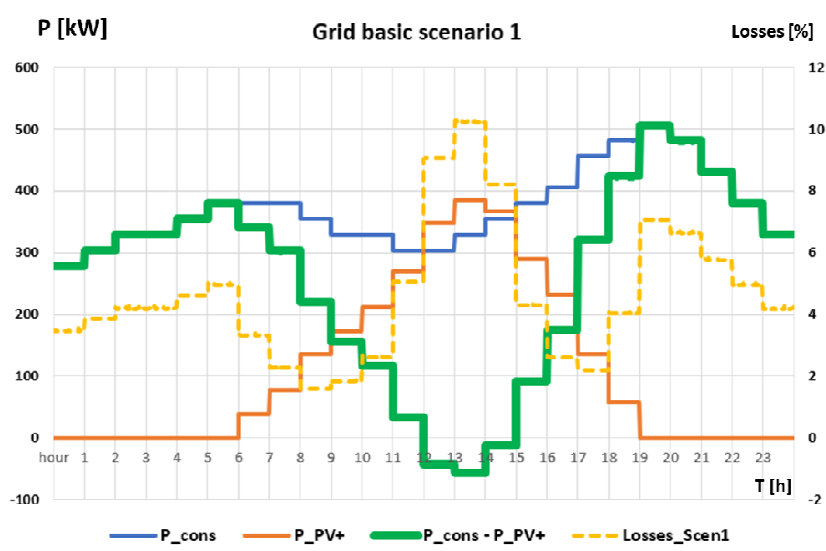

Fig. 10. Scenario 1 - Evolution of total consumed and produced power, their difference and the grid losses evolution

This behavior can be explained by analyzing the lines power flow evolution. Figure 11 shows the evolution of power flow over selected lines of the microgrid and reveals that the low losses occur in the period when some of the lines change the sign of the power, thus having for a certain period a very small power circulation, as it can be seen for lines N5-N6, N6N9 and N9-N10.

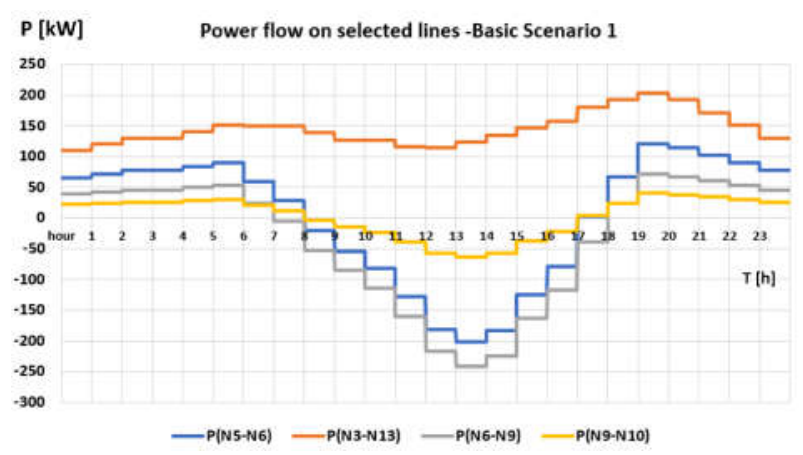

Fig. 11. Scenario 1 - Evolution of active power on selected lines of the microgrid

This situation suggests that storage acting as consumption and production facility at the right moments of the day may bring low power flow on longer time periods, thus bringing lower losses over the whole day.

\section{CONCLUSIONS}

The paper is analyzing microgrid behavior in a high RES penetration paradigm (more than $30 \%$ electricity RES-based generation occurs locally in the LV network over a day, around $100 \%$ consumption coverage during peak PV production), by using a specific microgrid topology and appropriate scenarios. Different topology measures based on looped LV networks and horizontal power exchange between the LV networks supplied by different transformers and presence of grid-side BESS are analyzed in terms of voltage level, MV/LV transformer power flow and grid losses. 
The assessment shows that new solutions in operating the $\mathrm{LV}$ microgrids and in using latest storage technologies are possible enablers for high RES penetration in LV networks, without the drawbacks of business as usual approach in the distribution network operation. Moreover, grid losses are lower in specific hours and over the whole day, as power flow becomes very low on some of the microgrid lines. Future work will consider batteries in a more realistic model, considering their losses and possible depreciation, while more dispersed grid-side storage will be also investigated.

\section{ACKNOWLEDGEMENTS}

The research work leading to these results has received funding from the European Union's Horizon 2020 research and innovation programme under grant agreement No 731155 Storage4Grid project.

\section{REFERENCES}

[1] Viyathukattuva M. A. M. Mansoor ; Phuong. H. Nguyen ; W. L. Kling, An integrated control for overvoltage mitigation in the distribution network, in Proc. of 2014 IEEE PES Innovative Smart Grid Technologies, Europe

[2] Walter Niederhuemer ; Roman Schwalbe, Increasing PV hosting capacity in LV grids with a probabilistic planning approach, in Proc. Of 2015 International Symposium on Smart Electric Distribution Systems and Technologies (EDST)

[3] Florian Javernik ; Elisabeth Hufnagl ; Maria Aigner ; Ernst Schmautzer, The compliant integration of (renewable) power sources in MV or LV grids and the impact on grid reliability, in Proc. Of 2015 International Conference on Renewable Energy Research and Applications (ICRERA)

[4] A.N.M.M. Haque ; D.S. Shafiullah ; P.H. Nguyen ; F.W. Bliek, Real-time congestion management in active distribution network based on dynamic thermal overloading cost, in Proc. of 2016 Power Systems Computation Conference (PSCC)

[5] Ruben Sanchez; Florin Iov ; Mohammed Kemal ; Maria Stefan ; Rasmus Olsen, Observability of low voltage grids: Actual DSOs challenges and research questions, Proceedigs of 2017 52nd International Universities Power Engineering Conference (UPEC)

[6] Angelos I. Nousdilis ; Grigoris K. Papagiannis ; Georgios C. Christoforidis, Investigating the impact of decentralized energy storage systems in active low-voltage distribution networks, in Proc. of 2017 52nd International Universities Power Engineering Conference (UPEC)

[7] M. Sanduleac, M. Albu, D. Stanescu, C.Stanescu, Prosumers optimally adapted to local load. Rationale and benefits for the grid, Modern Power Systems 2019 Cluj Napoca, Romania, May 21-23, 2019.
[8] Albu, Mihaela; Sanduleac, Mihai.; Dorel, Stanescu; Carmen, Stanescu 'Meter Accuracy in Renewable Energy Sources-based Prosumer Nodes' Proc. of the IEEE 7th International Conference on Modern Power Systems (MPS 2017), Cluj Napoca, Romania, ID 34, June 2017.

[9] Romanian Energy Regulatory Authority (ANRE) Order no. 208 of 14.12.2018 approving the technical norm on the technical requirements to connect power-generating modules, power plants modules and offshore power park modules to public electrical grids.

[10] Sanduleac, M.; Ciornei, Irina; Albu, Mihaela; Toma, L; Sturzeanu, Marta; Martins, JF, 'Resilient Prosumer Scenario in a Changing Regulatory Environment-The UniRCon Solution', Energies 2017, 10(7), 857.

[11] Mihai Sanduleac, Mihaela Albu, Lucian Toma, João Martins, Anabela Gonçalves Pronto, Vasco Delgado-Gomes, 2017, Hybrid AC and DC Smart home resilient Architecture Transforming prosumers in UniRCons - Proc. of ICE 2017 IEEE TMC Europe Conference, July 2017

[12] DIRECTIVE (EU) 2018/2001 OF THE EUROPEAN PARLIAMENT AND OF THE COUNCIL of 11 December 2018 on the promotion of the use of energy from renewable sources

[13] Law 184 from 18/07/2018, https://drive.google.com/file/d 11j8MYafWN7MoaSUmawShFgUhDDnrnNwR/view

[14] OpenDSS website: http://smartgrid.epri.com/SimulationTool.aspx, accessed 10.06.2019

[15] OpenDSS repository: https://sourceforge.net/projects/electricdss/ accessed 10.06.2019

[16] OpenDSS documentation: https://sourceforge.net/projects/ electricdss/files /OpenDSS/, accessed 10.06.2019

[17] Nagarajan, A., Ayyanar, R., Dynamic analysis of distribution systems with high penetration of PV generators using differential algebraic equations in OpenDSS, 2014 North American Power Symposium (NAPS), 7-9 Sept. 2014, Pullman, WA, USA (ieeexplore file: 06965355.pdf)

[18] Meghasai, Monger, S., Vega, R., Krishnaswami, H., Simulation of smart functionalities of photovoltaic inverters by interfacing OpenDSS and Matlab, 2015 IEEE 16th Workshop on Control and Modeling for Power Electronics (COMPEL), 12-15 July 2015, Vancouver, BC, Canada (ieeexplore file: 07236443.pdf)

[19] Montenegro, D., Hernandez, M., Ramos, G. A., Real time OpenDSS framework for distribution systems simulation and analysis, 2012 Sixth IEEE/PES Transmission and Distribution: Latin America Conference and Exposition (T\&D-LA), 3-5 Sept. 2012, Montevideo, Uruguay (ieeexplore file: 06319069.pdf)

[20] GridMonk repository: https://github.com/MihSand/GridMonK

[21] Denholm, P.; O’Connell, M.; Brinkman, G.; Jorgenson, J. Overgeneration from Solar Energy in California. A Field Guide to the Duck Chart; NREL: Denver USA, 2015; pp. 1-46 